\title{
Anisotropic Flow of Charged Particles at High Transverse Momentum in 2.76 TeV Pb-Pb Collisions at the LHC from ALICE experiment
}

\author{
Anitha Nyatha*广 \\ Indian Institute of Technology Bombay \\ E-mail: anithaiitb@gmail.com
}

\begin{abstract}
Anisotropic flow develops at early times of heavy-ion collision and is a powerful tool to understand the collision dynamics. We report on measurements of anisotropic flow components $v_{2}, v_{3}$, and $v_{4}$ for unidentified charged particles, pions and protons in $\mathrm{Pb}-\mathrm{Pb}$ collisions at $\sqrt{s_{N N}}=2.76$ $\mathrm{TeV}$ recorded by the ALICE experiment at the LHC. $v_{2}$ is presented for a wide range of particle transverse momentum up to $p_{T}=20 \mathrm{GeV} / c$. The particle $v_{2}$ is finite, positive and approximately constant for $p_{T}>8 \mathrm{GeV} / c$. The proton $v_{2}$ is higher than that of the pion up to about $p_{T}=8$ $\mathrm{GeV} / c$.
\end{abstract}

36th International Conference on High Energy Physics,

July 4-11, 2012

Melbourne, Australia

*Speaker.

${ }^{\dagger}$ On behalf of the ALICE Collaboration 


\section{Introduction}

The main goal of heavy-ion physics is to study the new phase of matter called Quark Gluon Plasma, predicted by Quantum Chromodynamics to exist at high energy density. An important observable used to characterize its properties is the anisotropic flow [1]. In non-central heavy-ion collisions, the geometrical overlap region, where the nuclei intersect and where initial interactions occur, is not isotropic. This initial spatial asymmetry is converted via multiple interactions into momentum-space anisotropy, leading more particle flow in the reaction plane. The reaction plane is spanned by the beam axis and the impact parameter. The anisotropic flow is characterized by the Fourier decomposition of azimuthal distribution of particles w.r.t the reaction plane.

$$
\begin{gathered}
E \frac{d^{3} N}{d^{3} P}=\frac{1}{2 \pi} \frac{d^{2} N}{p_{\mathrm{T}} d p_{\mathrm{T}} d y}\left(1+\sum_{n=1}^{\infty} 2 v_{n} \cos \left[n\left(\phi-\psi_{n}\right)\right]\right) \\
v_{n}\left(p_{T}, \eta\right)=\left\langle\cos \left[n\left(\phi-\psi_{n}\right)\right]\right\rangle
\end{gathered}
$$

where $p_{T}, \eta$, and $\phi$ are the particle's transverse momentum, pseudorapidity, and the azimuthal angle, respectively, and $\psi_{n}$ is the $n$-th harmonic symmetry plane angle. The elliptic flow $v_{2}$, is the second harmonic coefficient and the largest coefficient in midcentral collisions [2]. The large elliptic flow observed at RHIC showed that the matter created in heavy ion collisions behaves as a nearly perfect fluid whose constituent particles interact very strongly [3]. The ALICE Collaboration reported that, compared to RHIC energies the differential elliptic flow $\left(0.2<p_{T}<5 \mathrm{GeV} / c\right)$ does not change within uncertainties at LHC energies, but the integrated elliptic flow increases by about $30 \%$ [4]. In this study we extend the measurement of inclusive charged particles anisotropic flow out to $p_{T}=20 \mathrm{GeV} / \mathrm{c}$ and for protons and charged pions ${ }^{1}$ out to $p_{T}=16 \mathrm{GeV} / c$. We also present quadrangular flow coefficient, $v_{4}$, measured with respect to the second $\left(\psi_{2}\right)$ and fourth $\left(\psi_{4}\right)$ harmonic symmetry planes.

\section{Data taking and results}

The data recorded by the ALICE experiment [5] during the November-December 2010 LHC heavyion runs were used for this study. There are 18 sub- detectors in ALICE experiment, as shown in Figure.1, from which we used The Time Projection Chamber (TPC) for charged particle tracking and identification, and two VZERO detectors for centrality selection and the event plane determination. The event sample was collected with a dedicated minimum bias trigger. In order to remove beam induced background events, an offline event selection is applied. Furthermore, only events with a reconstructed primary vertex within $\pm 10 \mathrm{~cm}$ along the beam axis are selected for this analysis. Such a selection leads to around 10 million events. Only charged particles with $p_{T}>0.2$ $\mathrm{GeV} / \mathrm{c}$ are selected in the pseudorapidity range $|\eta|<0.8$. A Glauber model fit to the distribution of the summed amplitudes in the VZERO scintillator detectors was used to estimate the centrality of the collision [6].

\footnotetext{
${ }^{1}$ In the following, we use "pions" and "protons" to mean the sum of particles and anti-particles, unless mentioned otherwise.
} 


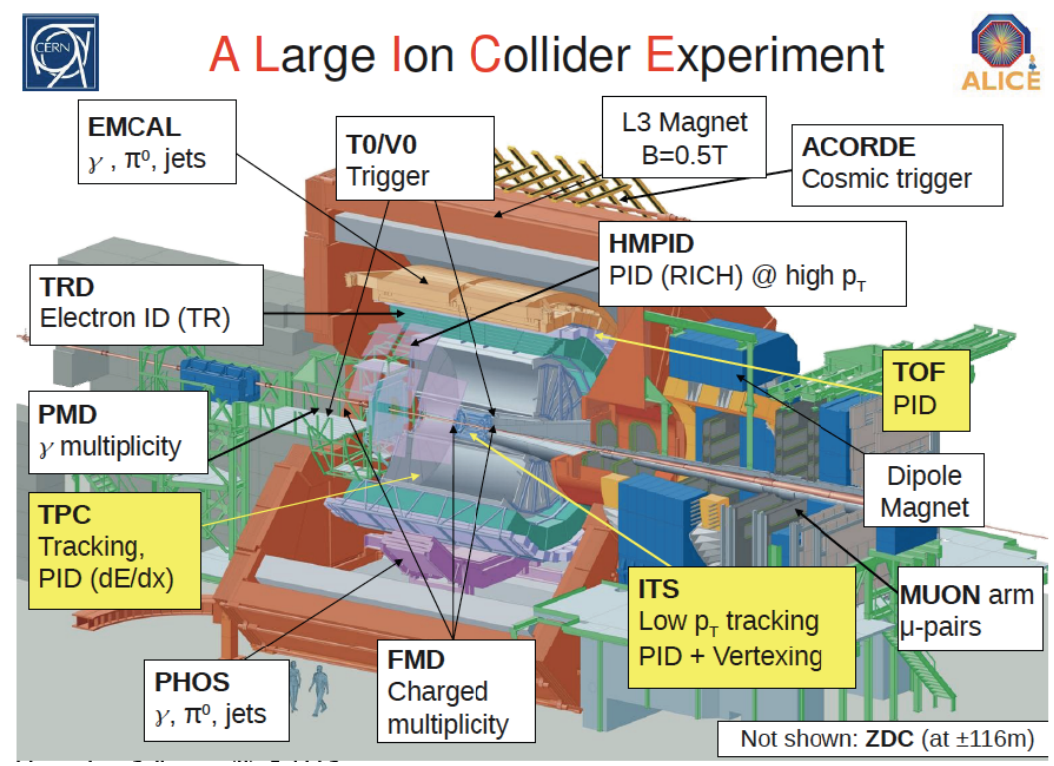

Figure 1: Schematic layout of ALICE experiment

Anisotropic flow was estimated with event plane $\left(v_{n}\{\mathrm{EP}\}\right)$ and four particle correlations ( $v_{2}\{4\}$ ) methods. The event plane was determined from VZERO signals.

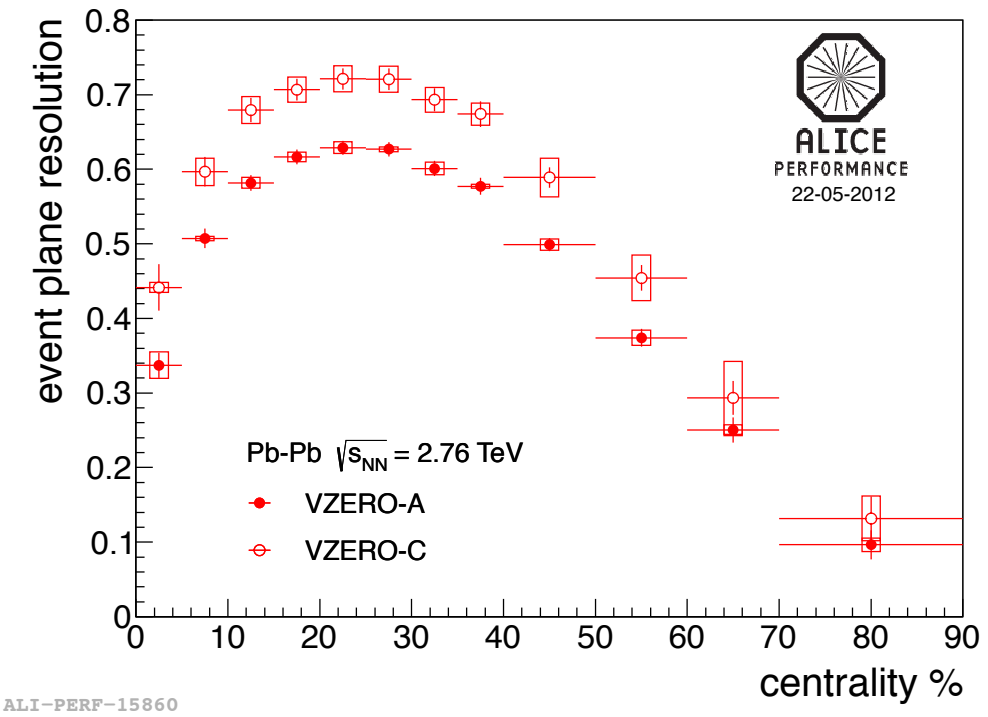

Figure 2: Event Plane resolution determined from two VZERO detectors

Figure. 2 shows the reaction plane resolution of VZERO detectors vs. centrallity. The detailed description of the methods and notation are given in reference [7].

The non-flow contribution in the event plane method is suppressed by using large rapidity gap of $|\Delta \eta|>2$ provided by VZERO detectors. Figure. 3 presents the differential flow components $v_{n}\left(p_{T}\right)$ for different centrality classes from $v_{2}\{\mathrm{EP},|\Delta \eta|>2.0\}, v_{2}\{4\}, v_{3}\{\mathrm{EP},|\Delta \eta|>2.0\}, v_{4 \psi_{4}}\{\mathrm{EP}$, 
$|\Delta \eta|>2.0\}, v_{4 \psi_{2}}\{\mathrm{EP},|\Delta \eta|>2.0\}$ methods. The results differ as expected since the methods have different sensitivity to flow fluctuations.

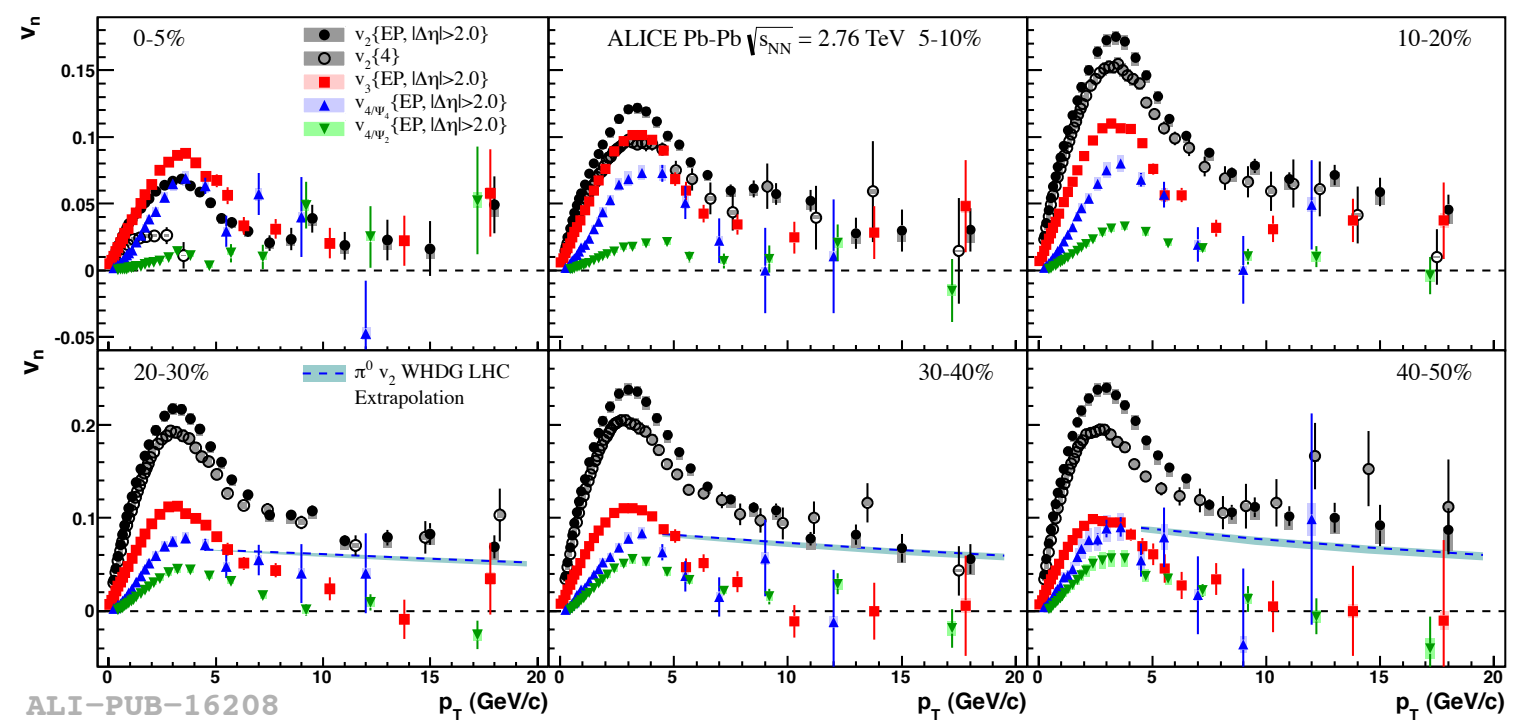

Figure 3: charged particle $v_{n}$ vs. $p_{T}$ in defferent centrality classes obtained with different methods [9]

$v_{2}$ at high transverse momentum $\left(p_{T}>8 \mathrm{GeV} / c\right)$ is finite and positive; its value increase from central to mid-peripheral events. $v_{2}\{\mathrm{EP}\}$ for $p_{T}>10 \mathrm{GeV} / c$ is reproduced by WHDG model calculations [8]. $v_{3}$ is smaller than $v_{2}$ in magnitude ( except for 0-5\% centrality) and exhibits much weaker centrality dependence. $v_{4 \psi_{4}}$ does not depend strongly on centrality compared to $v_{4} \psi_{2} . v_{4}$ at high $p_{T}\left(p_{T}>8 \mathrm{GeV} / c\right)$ is consistent with zero within relatively large uncertainties. 
Identified particle flow vs. $p_{T}$ allows to study the mechanism of particle production at different momentum scales.It gives insight into the hydrodynamical behavior of the created matter and can help to determine the region where the coalescence picture appears to be important. In ALICE, the charged particle identification (PID) for $p_{T}>3 \mathrm{GeV} / c$ can be done by employing the ionization energy loss, $\mathrm{dE} / \mathrm{dx}$, in the TPC. The TPC PID is based on the observable $\Delta \pi=d E / d x-\langle d E / d x\rangle_{\pi}$, where $\langle d E / d x\rangle_{\pi}$ is the expected energy loss for a pion. Particles with $1<\Delta \pi<7$ and $-25<$ $\Delta \pi<-14$ were identified as pions and (anti)protons, respectively. The estimated contamination was found to be below $1 \%$ for pions and below $15 \%$ for protons, respectively, in the measured $p_{T}$ range. Figure. 4 shows the charged pion and proton $v_{n}$ for $10-50 \%$ centrality measured with $v_{2}\{\mathrm{EP},|\Delta \eta|>2\}$ method. The proton $v_{2}$ and $v_{3}$ is higher than that of the pion out to at least $p_{T}=8$ $\mathrm{GeV} / c$, while the results start to overlap within systematic uncertainties for $p_{T}>8 \mathrm{GeV} / c$. Charged pion $v_{2}$ is reproduced by WHDG $\pi^{0}$ predictions for $p_{T}>7 \mathrm{GeV} / c$. Also the results are similar to that of neutral pions measured by PHENIX [10]

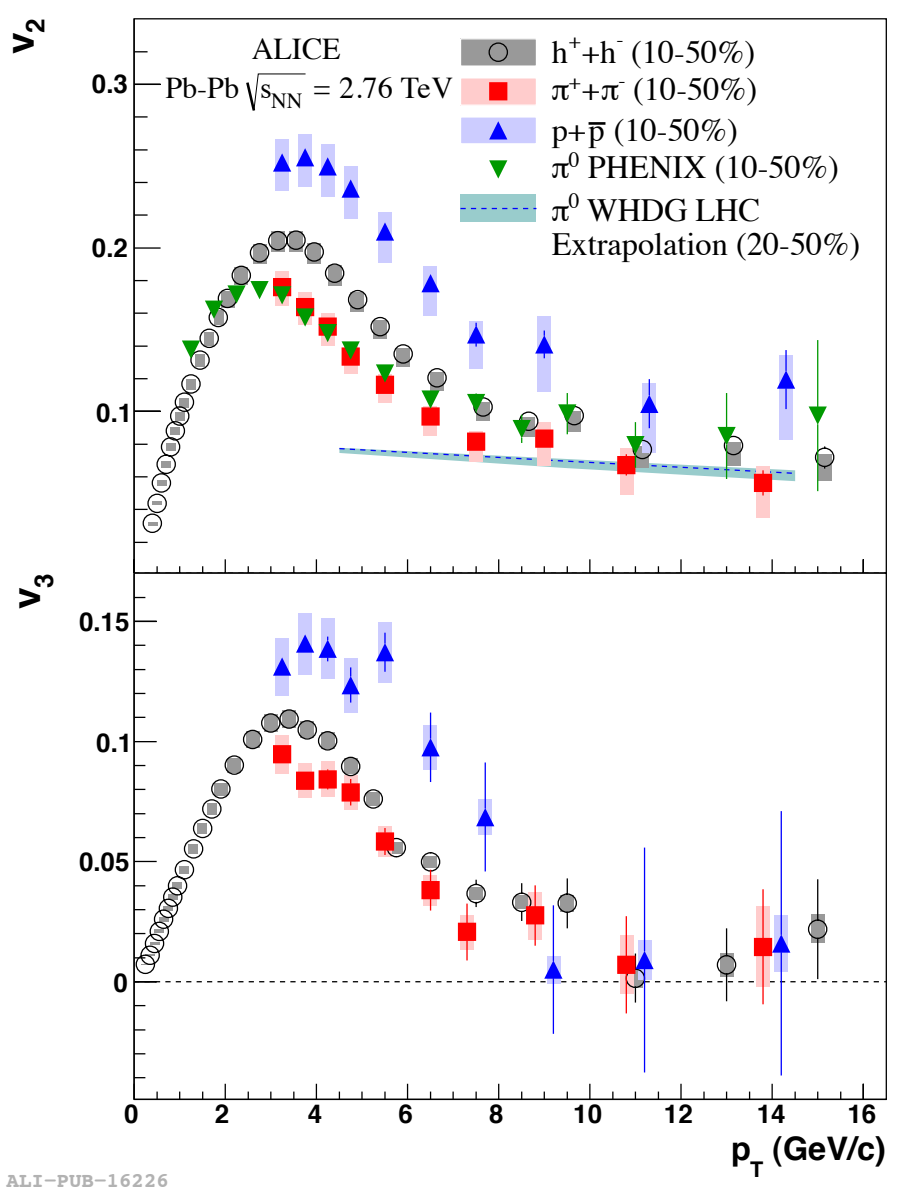

Figure 4: proton and pion $v_{2}$ and $v_{3}[9]$ 


\section{Summary}

Unidentified charged particle, proton and pion $v_{2}$ and $v_{3}$ have been measured up to $p_{T}=20$ $\mathrm{GeV} / c$. We found that unidentified charged particle $v_{2}$ is finite, positive and approximately constant at $p_{T}>8 \mathrm{GeV} / c$. Proton $v_{2}$ is higher than that of the pion up to about $p_{T}=8 \mathrm{GeV} / c$.

\section{References}

[1] J. Y. Ollitrault, Phys. Rev. D 46, 229 (1992).

[2] S. Voloshin and Y. Zhang, Z. Phys. C 70, 665 (1996) [arXiv:hep-ph/9407282].

[3] J. Adams et al. [STAR Collaboration], Nucl. Phys. A 757, 102 (2005).

[4] K. Aamodt et al. [The ALICE Collaboration], Phys. Rev. Lett. 105, 252302 (2010).

[5] K. Aamodt et al. [ALICE Collaboration], JINST 3, S08002 (2008).

[6] A. Toia, these proceedings.

[7] S. A. Voloshin, A. M. Poskanzer and R. Snellings, in Landolt-Boernstein, Relativistic Heavy Ion Physics, Vol. 1/23 (Springer-Verlag, 2010), p 5-54.

[8] W. A. Horowitz, M. Gyulassy, J. Phys. G 38, 124114 (2011).

[9] arXiv:1205.5761

[10] arXiv:1203.2644 